\title{
BMSC-derived exosomes from congenital polydactyly tissue alleviate osteoarthritis by promoting chondrocyte proliferation
}

\author{
Xinghua Zhou ${ }^{1,2}$, Hansi Liang ${ }^{3}$, Xiaohan $\mathrm{Hu}^{1}$, JinNan An ${ }^{4}$, Sisi Ding ${ }^{3}$, Shuichang $\mathrm{Yu}^{2}$, Cuiping Liu ${ }^{3}$, Fang Li ${ }^{2}$ and
} Yunyun $\mathrm{Xu}^{1}$

\begin{abstract}
In the past decade, mesenchymal stem cells (MSCs) have been widely used for the treatment of osteoarthritis $(\mathrm{OA})$, and exosomes may play a major role. Here, we acquired a special kind of MSCs from the bone marrow of surgically resected tissue from the hand of a patient with polydactyly. Experiments were focused on the role of polydactyly bone marrow-derived MSCs (pBMSCs) in osteoarthritis. The results showed that the pBMSCs had a greater ability than the BMSCs to differentiate into chondrocytes. Mechanistically, the expression of BMP4 was significantly higher in the pBMSCs than it was in the BMSCs. Furthermore, we showed that the migration and proliferation of chondrocytes were stimulated by exosomes secreted by pBMSC (pBMSC-EXOs). Notably, the downregulation of BMP4 in pBMSCs by siRNA inhibited both the chondrogenic differentiation potential of the MSCs and the function of the chondrocytes. In addition, the injection of PBMSC-EXOs and BMSC-EXOs attenuated OA in an OA mouse model, but the PBMSC-EXOs had a superior therapeutic effect compared with that of the BMSC-EXOs. Taken together, the data indicate that pBMSCs have greater ability to differentiate into chondrocytes and regulate chondrocyte formation through BMP4 signaling. Therefore, pBMSC-EXOs may represent a novel treatment for $O A$.
\end{abstract}

\section{Introduction}

Osteoarthritis (OA) is a chronic joint disease characterized by degeneration, destruction, and osteogenesis of joint cartilage. It is a "total joint disease"; that is, all the tissues in the joint are involved in the pathogenesis of $\mathrm{OA}^{1,2}$. The incidence of $\mathrm{OA}$ is closely related to the advancing age, and the prevalence rate of $\mathrm{OA}$ in females is significantly higher than that it is in males $^{3}$. The pathogenesis of OA is complex and has a heritable

\footnotetext{
Correspondence: Fang Li (lif@suda.edu.cn) or Yunyun Xu (rdxyy@163.com) ${ }^{1}$ Institute of Pediatrics, Children's Hospital of Soochow University, Suzhou, Jiangsu, China

2Department of Human Anatomy, Histology and Embryology, School of Biology and Basic Medical Sciences, Soochow University, Suzhou, Jiangsu, China

Full list of author information is available at the end of the article These authors contributed equally: Xinghua Zhou, Hansi Liang Edited by Alessandro Rufini
}

tendency ${ }^{4}$. In general, it is believed that, due to the interaction of mechanical and biological factors, imbalances in articular chondrocytes, the extracellular matrix (ECM) and the extent of subchondral bone synthesis and degradation are the main reasons for the development of $\mathrm{OA}^{5}$. Currently, nondrug therapy, drug therapy, and surgical treatment in the clinic do not fundamentally delay the progressive degeneration of articular cartilage during OA. In view of this, a new cellbased treatment of $\mathrm{OA}$ has attracted increasing attention.

Mesenchymal stem cells (MSCs), with the potential of self-renewal and directed differentiation, can repair cartilage tissue and inhibit the secretion of inflammatory factors by chondrocytes. Furthermore, MSCs can be directed to differentiate into chondrocytes in vivo, which provides a theoretical basis for the treatment of

\section{(c) The Author(s) 2020}

(c) (i) Open Access This article is licensed under a Creative Commons Attribution 4.0 International License, which permits use, sharing, adaptation, distribution and reproduction cc) in any medium or format, as long as you give appropriate credit to the original author(s) and the source, provide a link to the Creative Commons license, and indicate if changes were made. The images or other third party material in this article are included in the article's Creative Commons license, unless indicated otherwise in a credit line to the material. If material is not included in the article's Creative Commons license and your intended use is not permitted by statutory regulation or exceeds the permitted use, you will need to obtain permission directly from the copyright holder. To view a copy of this license, visit http://creativecommons.org/licenses/by/4.0/. 
OA by MSCs. Moreover, a series of studies confirmed that MSCs can effectively treat OA from different aspects $^{6-8}$. Clinical application has preliminarily proven that MSCs may be the best method to treat traumatic bone and cartilage defects ${ }^{9}$. However, the therapeutic effects of BMSCs are not ideal ${ }^{10}$. In addition, the risk of tumor formation, ethical issues, and transplant rejection remain barriers to further stem cell studies ${ }^{11}$. Besides, unified standards for the isolation methods of stem cells and the optimal storage conditions needed to maintain cell viability are lacking, which severely limits the further clinical application of stem cell therapy. Consequently, new strategies to overcome these disadvantages are still needed.

In recent years, research on the paracrine effect of stem cells in the process of tissue repair has attracted considerable attention. Exosomes are tiny vesicles released into the ECM upon the fusion of multivesicular bodies with the cytoplasmic side of the plasma membrane. Exosomes are shaped like discs and have a diameter of $30-150 \mathrm{~nm}^{12-14}$. In addition, exosomes contain a variety of proteins, lipids, and nucleic acids with important functions and can transmit these specific components as signal molecules to both nearby and distant cells, affecting the function of recipient cells. In recent years, exosomes from stem cells have been shown to be involved in the regeneration of myocardial tissue ${ }^{15,16}$, lung tissue ${ }^{17,18}$, the retina ${ }^{19}$, central nervous system ${ }^{20}$, kidney tissue ${ }^{21}$, and pancreatic tissue ${ }^{22}$. Our previous studies of amniotic fluid stem cell (AFS) transplantation for skin injury repair have suggested that transplanted human AFS does not promote tissue repair by differentiating into the same type of cells in damaged tissue but by affecting the wound repair microenvironment through paracrine signaling that promotes the repair of damaged skin tissue $^{23}$, which is additional evidence showing that stem cell-derived exosomes may be an alternative to cell therapy.

Congenital polydactyly is manifested as a malformation of the hand, and surgical resection is currently the common treatment for this disease ${ }^{24}$. With informed consent, we obtained a special type of mesenchymal stem cells (pBMSCs) from bone marrow extracted from the soft tissue of surgically resected fingers. Subsequently, using immunophenotypic identification and functional analysis, we found that pBMSCs have greater capacity than BMSCs for in vitro amplification. Importantly, pBMSCs have a profound ability to differentiate into chondrocytes. In addition, the BMP4 pathway plays not only a regulatory role in the differentiation of pBMSCs into chondrocytes but also an important role in the promotion of the growth of chondrocytes through the secretion of pBMSC-derived exosomes (pBMSC-EXOs). Finally, pBMSC-EXOs injection into the joint cavity significantly improved cartilage injury in the knee of a collagenase-induced OA mouse model, as shown by the OARSI score. Here, our study suggests that pBMSC-EXOs, once considered "medical waste", can function as a treatment for OA when administered properly.

\section{Materials and methods \\ Primary cell cultures \\ pBMSCs and BMSCs}

Polydactyly tissue was obtained by surgical resection during treatment for congenital polydactyly disease. pBMSCs were collected from bone marrow aspirates of this polydactyly tissue, while BMSCs were collected from bone marrow aspirates of healthy adult donors. Written informed consent was obtained from all patients and donors. The method for BMSCs preparation was described previously ${ }^{25}$. Moreover, the collected cells were cultured in low-glucose Dulbecco's modified Eagle's medium (LG-DMEM) supplemented with $10 \%$ fetal bovine serum (FBS; Gibco, CA, USA).

\section{Chondrocytes}

The articular cartilage of limbs from the polydactyly tissue sample was separated under aseptic conditions, and then, the fascia and cartilage membrane surrounding the cartilage tissue were peeled off. Next, the cartilage tissue was cut into $\sim 0.5-\mathrm{mm}$ tissue blocks and washed three times with PBS containing penicillin and streptomycin. The cartilage tissue was digested for $1-2 \mathrm{~h}$ with $0.25 \%$ trypsin at $37^{\circ} \mathrm{C}$. After trypsin digestion, the cartilage tissue continued to be digested overnight with $0.02 \%$ type II collagenase at $37^{\circ} \mathrm{C}$, and finally, a single cell was isolated. The isolated chondrocytes were collected and cultured with DMEM/F12 containing 10\% FBS.

All the cells used were analyzed between passage 3 and 5. The use of the pBMSCs, BMSCs, and chondrocytes was approved by the Ethics Committee of Soochow University (approval No. SUDA20200707H01).

\section{Characterization of BMSCs and pBMSCs}

The immunophenotypes of the BMSCs and pBMSCs were determined by flow cytometry analysis. The cells were harvested and incubated with monoclonal antibodies against CD105, CD106, CD34, CD90, CD45, CD133, CD73, CD14, and HLA-DR or the isotype control (eBioscience) for $30 \mathrm{~min}$ at $4{ }^{\circ} \mathrm{C}$. Later, the surface antigens of the cells were analyzed using a Gallios flow cytometer and analyzed using Kaluza software (Beckman Coulter). 


\section{Trilineage differentiation of the BMSCs and pBMSCs Osteogenic differentiation}

For osteogenic differentiation, BMSCs and pBMSCs were seeded at a density of $5 \times 10^{3}$ cells $/ \mathrm{cm}^{2}$. Then, MSCs were cultured with LG-DMEM containing 10\% FBS, $0.1 \mathrm{mM}$ dexamethasone, $50 \mathrm{mM}$ vitamin $\mathrm{C}$, and $10 \mathrm{mM}$ glycerophosphate. After the cells grew to $60-70 \%$ confluence, the osteogenic induction medium was replaced every 3 days. After 2 weeks, the osteogenic differentiation of the BMSCs was analyzed by alizarin red staining. Mineral nodules appeared in the pBMSCs after 1 month in culture.

\section{Adipogenic differentiation}

For driving adipogenic differentiation, induction medium (DMEM containing 10\% FBS, $1 \mathrm{mM}$ dexamethasone, $200 \mathrm{mM}$ indomethacin, $0.5 \mathrm{mM}$ isobutylmethylxanthine and $10 \mathrm{mg} / \mathrm{mL}$ insulin) was added to the BMSCs and pBMSCs after the cells reached $100 \%$ confluence. The adipogenic induction medium was replaced every 3 days. After 2 weeks, the lipid accumulation in BMSCs was determined by oil red $\mathrm{O}$ staining, and the lipids appeared in the pBMSCs after 1 month.

\section{Chondrogenic differentiation}

For chondrogenic differentiation, the BMSCs and pBMSCs were seeded at a density of $5 \times 10^{5}$ cells/15$\mathrm{mL}$ conical tubes via centrifugation at $250 \times g$ for $5 \mathrm{~min}$. Then, the cells were induced with chondrogenic media (LG-DMEM containing $1 \% \mathrm{FBS}, 0.01 \mu \mathrm{g} / \mathrm{mL}$ TGF- $\beta 3, \quad 0.4 \mu \mathrm{g} / \mathrm{mL}$ dexamethasone, $0.05 \mu \mathrm{g} / \mathrm{mL} 2$ phospho-L-ascorbic acid, $0.04 \mathrm{mg} / \mathrm{mL}$ proline, and $1 \%$ insulin-transferrin-selenium solution (Thermo Fisher Scientific, Waltham, MA). The induction medium was replaced every 3 days. Cell pellets were maintained in culture for 7-21 days. Finally, the pellets were identified by paraffin embedding, sectioning, and toluidine blue staining.

\section{Quantitative reverse transcription PCR}

Total RNA was isolated using a NucleoSpin RNA kit (MACHEREY-NAGEL, Duren, Germany) and reverse transcribed with a PrimeScript Reverse Transcriptase kit (TaKaRa, Dalian, China). Next, quantitative reverse transcription PCR was performed using a HiScript II One Step qRT-PCR SYBR Green kit (Vazyme, Nanjing, China) on an iQ5 Real-time PCR Detection system (Bio-Rad, Hercules, CA, USA). Moreover, the relative gene expression was analyzed by the $2^{-\Delta \Delta C T}$ method. The primers for Acan, Sox9, CoL2A1, Foxc2, BMP4, CTNNB1, c-Myc, and Actin are listed in Table S1.

\section{Immunofluorescence}

After blocking with $5 \%$ bovine serum albumin, the BMSCs and pBMSCs were incubated overnight at $4{ }^{\circ} \mathrm{C}$ with primary antibodies: rabbit anti-human BMP4 (1:100, Abcam). Then, the cells were washed with PBS and incubated with HRP-conjugated secondary antibodies (Life Technologies, Duren, DE) for 30 min at room temperature. Finally, images were captured by fluorescence microscopy (Nikon Eclipse Ni, Tokyo, Japan) after the cell nuclei were stained with DAPI (SouthernBiotech, AL, USA).

\section{Isolation and identification of the BMSC-EXOs and pBMSC- EXOs}

Exosomes were isolated and purified from the supernatant of the pBMSCs and BMSCs according to an established protocol ${ }^{26,27}$. Upon reaching $80-90 \%$ confluency, the cells were washed with PBS, and the culture medium was changed to LG-DMEM without FBS. After culturing for $48 \mathrm{~h}$, the cultured supernatant was collected and centrifuged at $300 \times g$ for $10 \mathrm{~min}$ and then at $2000 \times g$ for $30 \mathrm{~min}$ at $4{ }^{\circ} \mathrm{C}$. Next, the supernatant was transferred to a new tube without disturbing the pellet. Finally, the exosomes were collected according to the protocol of a total exosome isolation kit (Invitrogen ${ }^{\mathrm{TM}}$, CA, USA).

The concentration and size distribution of the pBMSCEXOs and BMSC-EXOs were measured by ZetaView (Particle Metrix, Germany). The morphology of the exosomes was determined by observation via an FEI Tecnai G2 spirit transmission electron microscope (TEM; FEI, Eindhoven, the Netherlands). Measurements of the characteristic proteins CD9 (1:1000; Abcam, Cambridge, UK), CD63 (1:1000; Abcam), and TSG101 (1:1000; Santa Cruz, Dallas, TX, USA) in the exosomes were determined by western blotting.

\section{Coculture experiments}

The pBMSCs, BMSCs, and chondrocytes were cultured according to previous protocols. Coculture experiments were performed by seeding chondrocytes $\left(5 \times 10^{4}\right)$ with pBMSCs or BMSCs $\left(5 \times 10^{4}\right)$ in a six-well plate. Furthermore, chondrocytes $\left(5 \times 10^{4}\right)$ were seeded in the lower chamber, and pBMSCs or BMSCs $\left(5 \times 10^{4}\right)$ were seeded in the upper chamber of a six-well Transwell apparatus with a $0.4-\mu \mathrm{m}$ pore size (Corning Incorporated, NY, USA). After $48 \mathrm{~h}$, we evaluated the function of the BMSC-EXOs and pBMSC-EXOs in promoting the proliferation of chondrocytes by calculating the cell number.

\section{Wound-healing assay}

After being treated with pBMSC-EXOs or transduced with siRNA against BMP4 and the chondrocyte culture 
reached $90-100 \%$ confluence, a scratch was made in the cultured monolayer. Small interfering RNA targeting specific human BMP4 sites (si480 and si1318) and a negative control siRNA (siNC) were synthesized by GenePharma (Shanghai, China) (Table S2). Then, the cells were washed with PBS to remove detached cells. Images of the wound area were captured at different time points (24, 48, and $72 \mathrm{~h}$ ) by a microscope (Nikon Eclipse $\mathrm{Ti}$, Tokyo, Japan) connected to a Nikon camera using NISElements software.

\section{Migration assay}

Transwell Permeable Support (Corning, NY, USA) was used for the migration assay of chondrocytes with or without pBMSC-EXOs. Coculture experiments were performed by seeding chondrocytes $\left(5 \times 10^{4}\right)$ in the upper chamber and pBMSC-EXOs in the lower chamber of the system. After incubation for $24 \mathrm{~h}$, the migrated cells were stained with crystal violet and counted.

\section{Collagenase-induced OA model}

Six-week-old male C57BL/6J mice were anesthetized with sodium pentobarbital $(1 \%(\mathrm{~m} / \mathrm{v}), 8 \mathrm{~mL} / \mathrm{kg})$ and randomly distributed into four groups: control $(n=5)$, OA $(n=6)$, BMSC-EXOs treatment $(n=6)$, and pBMSCEXOs treatment $(n=6)$. After anesthesia, collagenase VII (Clostridium histolyticum, Sigma-Aldrich, USA) was used to create the OA model according to the described protocol $^{28}$. In the control group, the knee joints of the mice were injected with $10 \mu \mathrm{L}$ saline intra-articularly through the patellar ligament. In the other groups, $10 \mu \mathrm{L}$ of saline with collagenase was injected into the knee joints in the same way. For the therapeutic experiment, we injected saline, BMSC-EXOs, and pBMSC-EXOs into the OA, BMSC-EXOs, and pBMSC-EXOs groups, respectively, on days 7,14 , and 21 . The mice were euthanized for further histology analysis on day 28. All operations were performed under protocols approved by the Ethics Committee of Soochow University and the international guidelines for animal experimentation (approval No. SUDA20200707A02).

\section{Histology}

The mouse joints were isolated from the OA model and fixed with $10 \%$ buffered paraformaldehyde for $24 \mathrm{~h}$. Before being embedded in paraffin, mouse tibias were decalcified in 10\% EDTA ( $\mathrm{pH} 7.4$ ) according to a standard protocol ${ }^{29}$. Furthermore, the $5-\mu \mathrm{m}$-thick sagittal joint sections were stained with hematoxylin and eosin $(\mathrm{H} \& \mathrm{E})$ and safranin $\mathrm{O} /$ fast green. The severity of the cartilage destruction was evaluated based on the Osteoarthritis Research Society International (OARSI) scoring system ${ }^{30}$.

\section{Statistical analysis}

All statistical values were calculated by using SPSS v18 (SPSS, Chicago, IL, USA). Data are reported as the means \pm standard deviation. Two groups were compared by unpaired Student's $t$ test, while more than two groups were assessed through one-way analysis of variance followed by Bonferroni test. A $p$ value $<0.05$ was considered significant.

\section{Results \\ Characterization of the pBMSCs}

We cultured MSCs from polydactyly tissue and then identified their specific immunophenotype. The results showed that pBMSCs had an immunophenotype similar to that of BMSCs, with high expression of MSC surface markers CD105, CD166, CD90, and CD73, no expression of hematopoietic stem cell surface markers CD133 or CD34, and no expression of immune cell surface markers CD45 or HLA-DR (Fig. 1A, B). In addition to similar immunophenotypes, we compared the biological characteristics of pBMSCs with those of BMSCs, and the results of the growth curve showed that pBMSCs had higher capacity for in vitro amplification than did BMSCs (Fig. 1C).

\section{Greater ability of the pBMSCs to differentiate into chondrocytes}

We further analyzed the ability of pBMSCs to differentiate into bone, cartilage, and adipocytes. The results showed that BMSCs differentiate into adipocytes and bone cells in 14 days and pBMSCs differentiated into adipocytes and bone cells in 60 days, which showed that pBMSCs have a lower ability than BMSCs to differentiate into adipocytes and bone cells (Fig. 2A, B, D, E). However, the differentiation time of pBMSCs into chondrocytes was 7 days, significantly less time than the 14 days for the BMSCs to differentiate into chondrocytes (Fig. 2C, F). Thus, pBMSCs have a greater ability than BMSCs to differentiate into chondrocytes. Based on this finding, we further analyzed the changes in chondrogenic genes before and after the pBMSCs and BMSCs differentiated into chondrocytes. The results showed that 14 days after induction, the expression of Acan and CoL2A1 in the chondrocytes derived from the pBMSCs was significantly higher than that in the chondrocytes derived from the BMSCs (Fig. 2G).

\section{pBMSCs regulate chondrocyte formation through BMP4 signaling}

In the process of chondrocyte formation, BMP4, WNT/ $\beta$-catenin, and other signaling pathways play important roles. Therefore, we compared and analyzed the expression of Foxc2, Sox9, BMP4, CTNNB1, and c-myc in the 
A

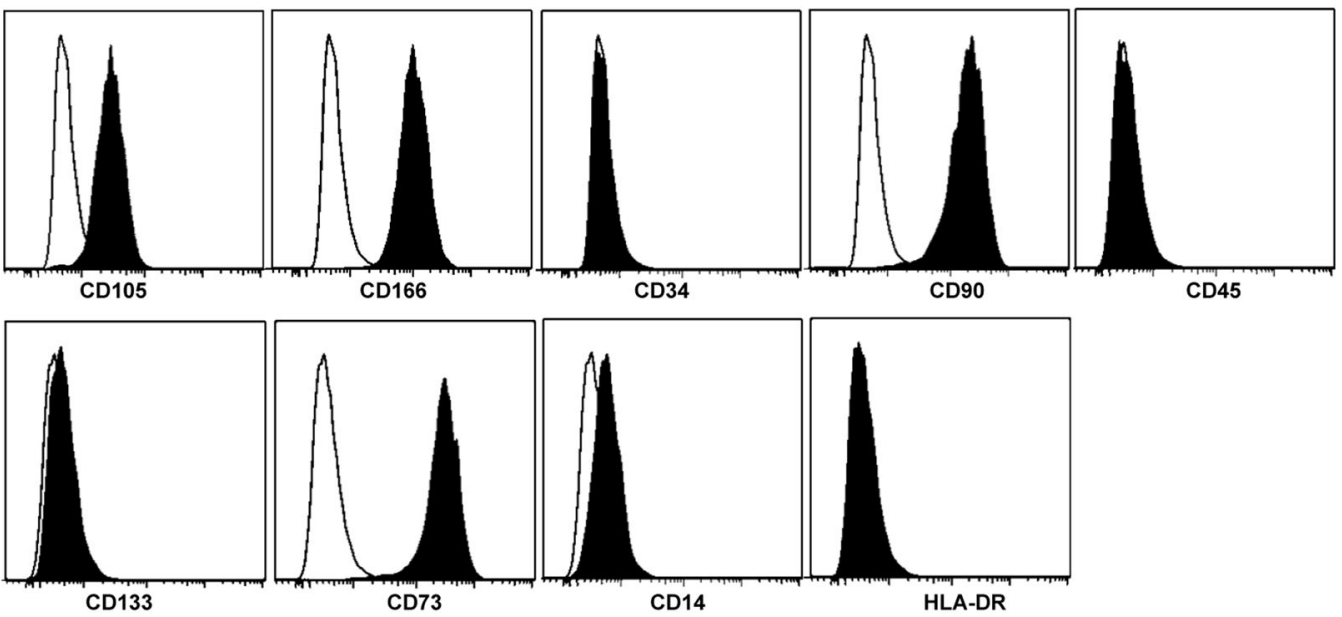

B
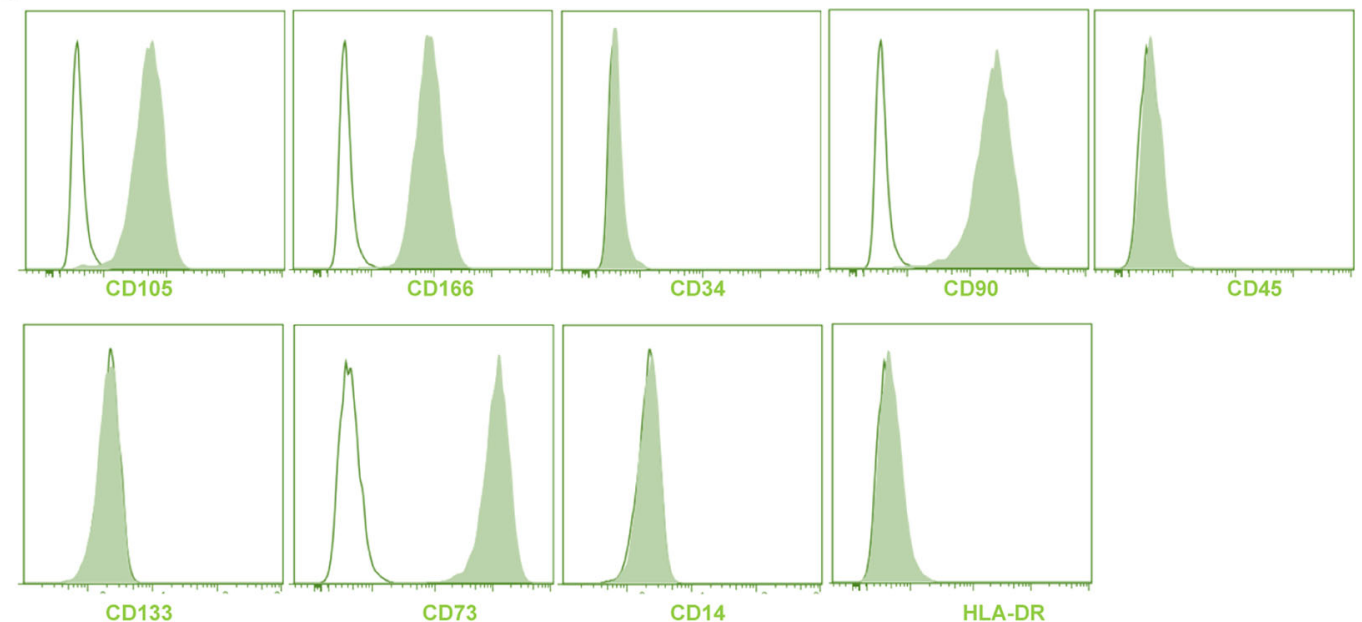

C

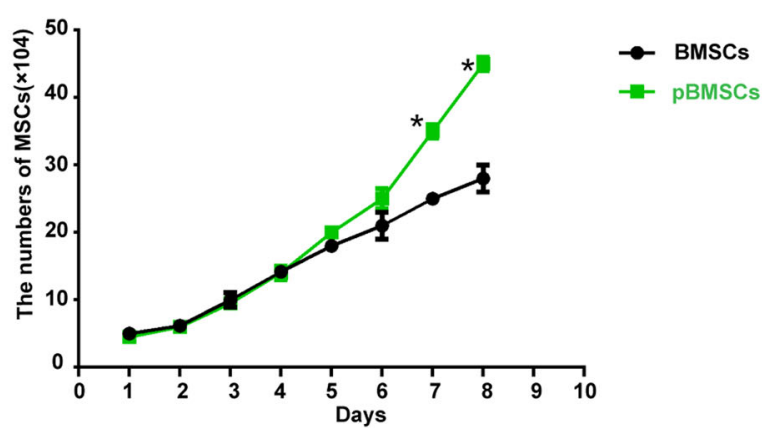

Fig. 1 Immune phenotypes and growth curves of the pBMSCs. Flow cytometric analysis of immune phenotypes of A MSCs from polydactyly tissue and $\mathbf{B}$ MSCs from bone marrow. The blank areas indicate the control, and the filled areas indicate the treated cells. C Growth curves of the pBMSCs and BMSCS cultured in vitro, ${ }^{*} P<0.05$.

pBMSCs and BMSCs. The results showed that the expression of BMP4 in the pBMSCs was significantly higher than it was in the BMSCs (Fig. 3A). In addition, the same expression pattern of BMP4 was observed at the protein level (Fig. 3B). Furthermore, after downregulation of BMP4 expression by siRNA (Fig. S1), the ability of the PBMSCs to undergo chondrogenic differentiation was inhibited (Fig. 3C). 

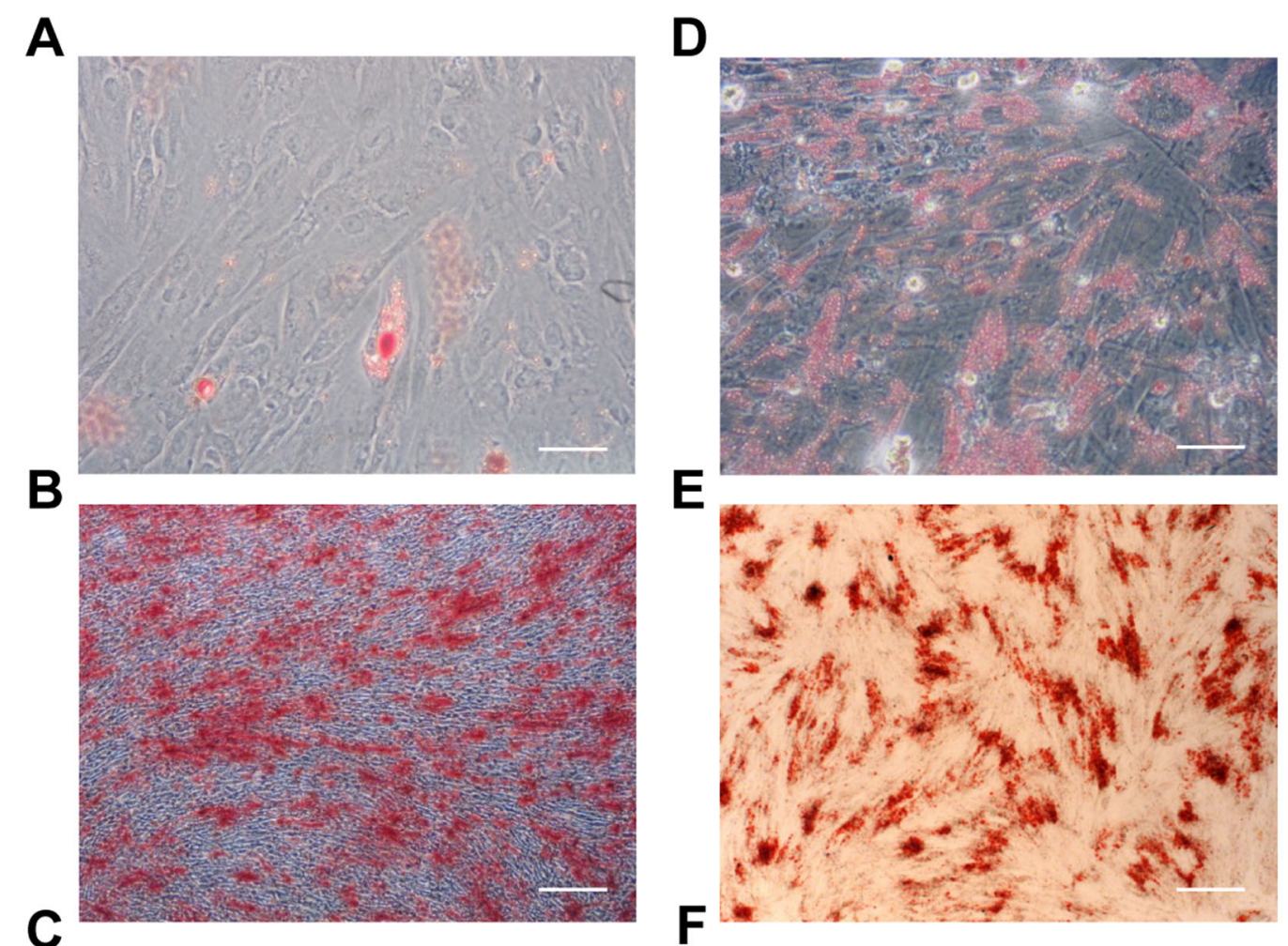

E
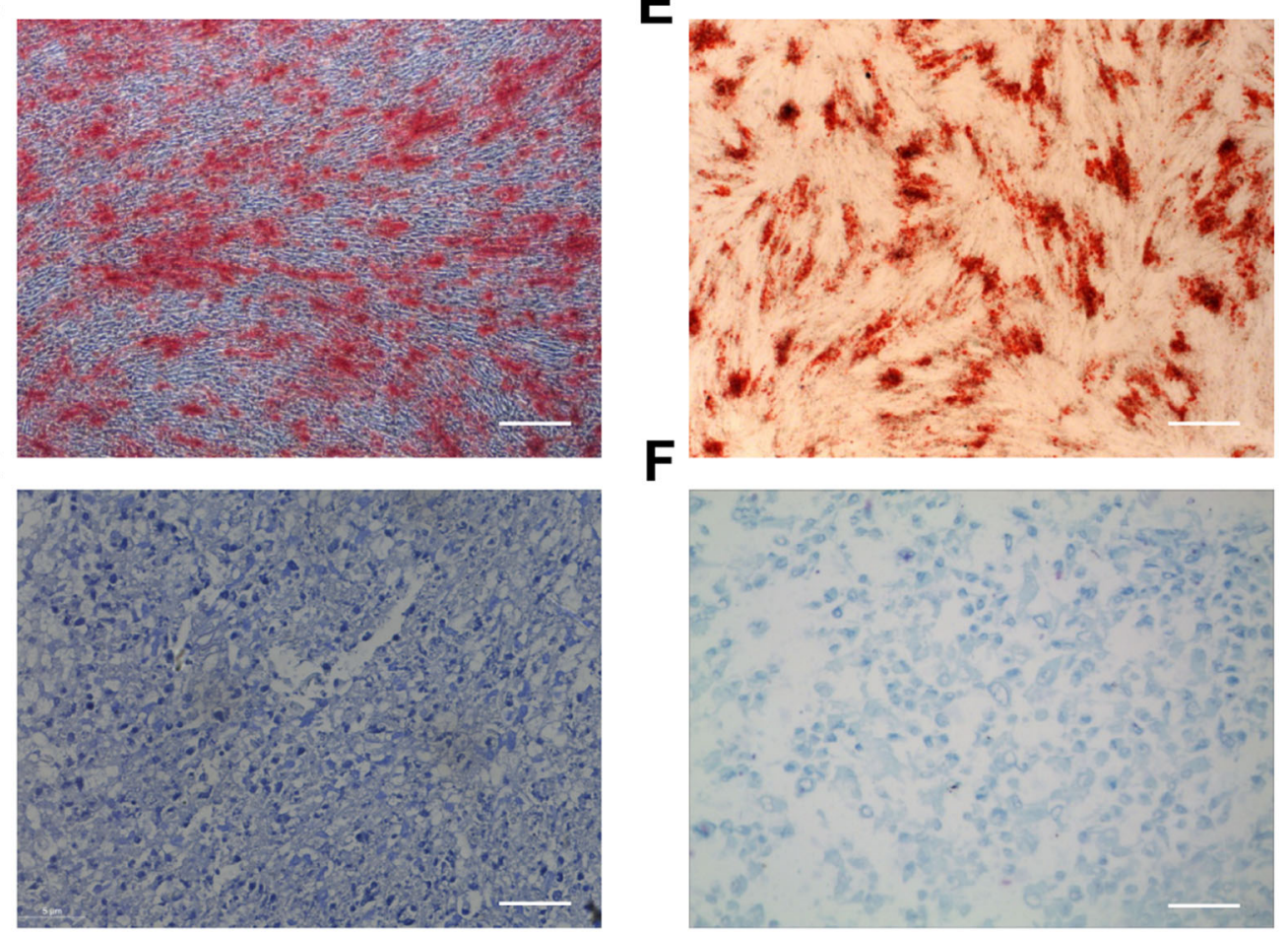

G

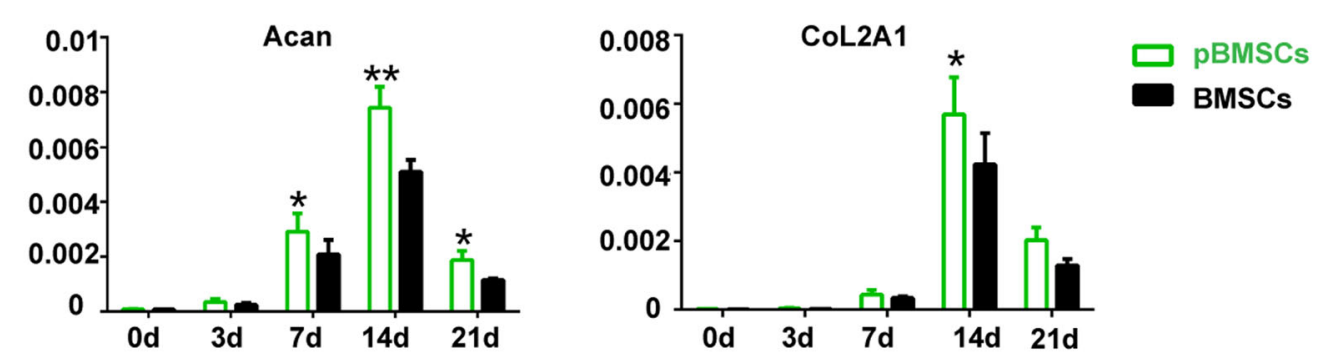

Fig. 2 The potential of the three-dimensional differentiation of the pBMSCs. A-C pBMSCs were purified from polydactyly bone marrow and then induced into adipose, osteoblast, and chondroblast cells. Scale bars, $40 \mu \mathrm{m}$. D-F BMSCs were purified from donor bone marrow and then induced into adipose, osteoblast, and chondroblast cells. Scale bars, $40 \mu \mathrm{m}$. G Real-time PCR analysis of Acan and CoL2A1 mRNA expression in the pBMSCs at different time points and BMSCs after induction into chondroblast cells. 


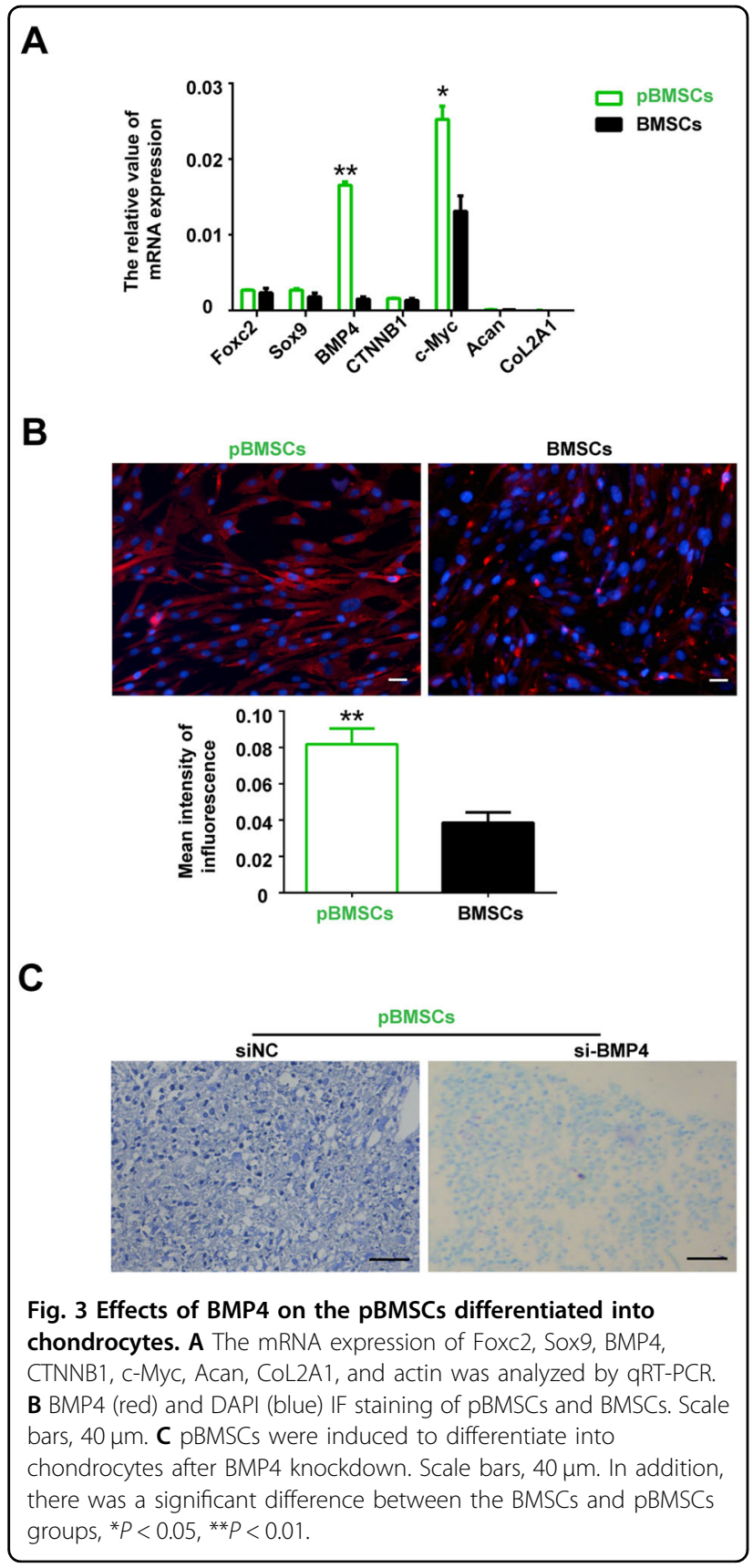

The exosomes secreted by the pBMSCs support the growth of chondrocytes in vitro

The pBMSCs are capable of readily differentiating into chondrocytes, but do they influence the growth of chondrocytes in vitro? To answer this question, we cocultured pBMSCs with chondrocytes to monitor the proliferation of the chondrocytes. The results showed that pBMSCs promoted chondrocyte proliferation in vitro (Fig. 4A). Compared to Microvesicles from pBMSCs, the exosomes derived from pBMSCs were mainly involved in the regulation of chondrocyte biological behaviors (Fig. S2). We isolated exosomes from the supernatant of the pBMSCs cultures by multiple overspeed centrifugation combined with a filtration method. Subsequently, we identified pBMSC-EXOs that expressed the exosome-specific proteins CD63 and CD9 (Fig. 4B). In addition, the typical $30-150 \mathrm{~nm}$ vesicle structure of the exosomes was observed with a transmission electron microscope (Fig. 4C). Especially, we found that $\mathrm{pBMSC}$-EXOs were ingested by chondrocytes (Fig. S3), which promoted chondrocyte migration and proliferation of chondrocytes (Fig. 4D, E). Compared with pBMSC-EXOs, pBMSC-siBMP4derived exosomes inhibited the migration and proliferation of chondrocytes, which demonstrated that BMP4 signaling also plays an important role in promoting chondrocyte proliferation (Fig. 5 and Fig. S4). In addition, pBMSC-EXOs had no effects on fibroblastlike synoviocytes (FLSs) in the proliferated synovial tissue (Figs. S5 and S6), while FLSs were involved in the destruction of articular bone and cartilage during the progression of OA.

\section{Exosomes secreted by the pBMSCs for the treatment of OA}

We constructed an OA mouse model induced by collagenase and then treated it with pBMSC-EXOs injected into the articular cavity (Fig. 6A). After 28 days of treatment, knee joint specimens (tibial plateau) were collected from the mice. The results of H\&E staining and safranin $\mathrm{O} /$ fast green staining confirmed that pBMSC-EXOs treatment reduced the degree of cartilage damage in the OA model (Fig. 6B, C). Moreover, the OARSI scores were significantly higher for the OA model group and significantly lower for the pBMSCEXOs treatment group than it was for the normal control group (Fig. 6D and Table S3). These results showed that pBMSC-EXOs can alleviate the cartilage damage induced by $\mathrm{OA}$.

\section{Discussion}

In the study, in addition to an immunophenotype similar, pBMSCs isolated from the specimens surgically excised from of children with polydactyly exhibited a greater ability than the BMSCs to proliferate and differentiate into chondrocytes. Furthermore, pBMSCderived exosomes not only promoted the proliferation and migration of chondrocytes in vitro but also reduced the degree of joint injury in the OA model mice.

As pluripotent stem cells, BMSCs have played important roles in repairing and mitigating tissue damage in a variety of tissue damage models ${ }^{31-33}$. However, when articular cartilage is injured, spontaneous repair is very difficult because the perichondrium covering the articular cartilage surface is absent, bone progenitor cells are lost 
A

D
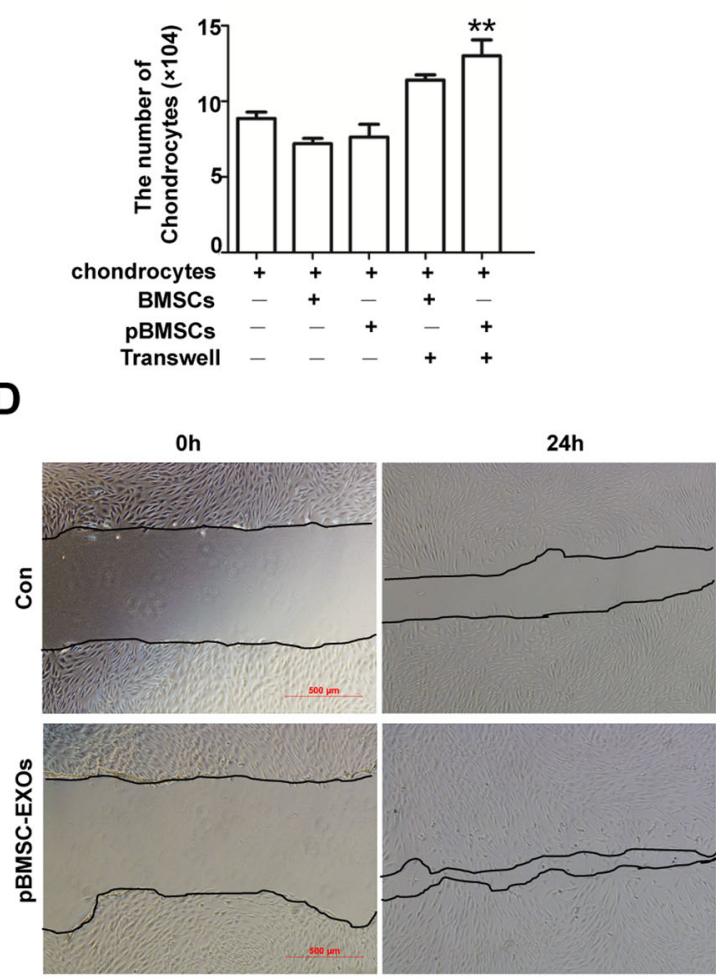

pBMSCs/ BMSCs

Chondrocytes

C

B
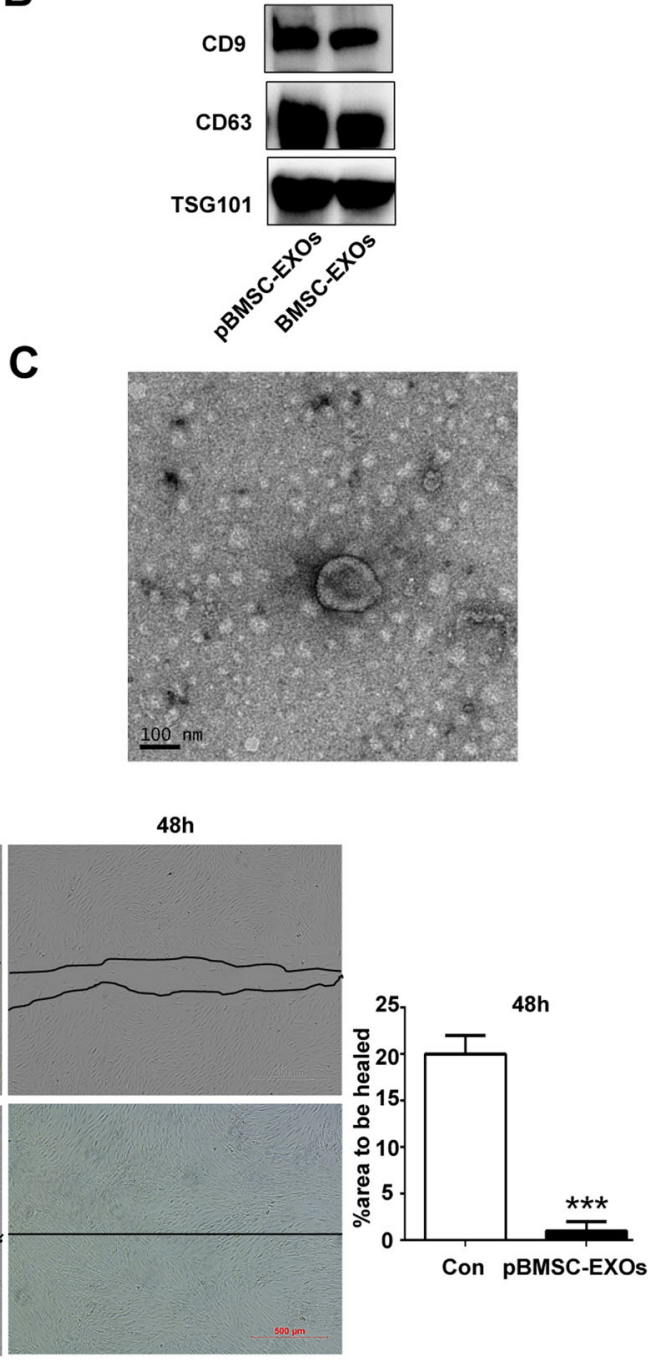

$\mathbf{E}$
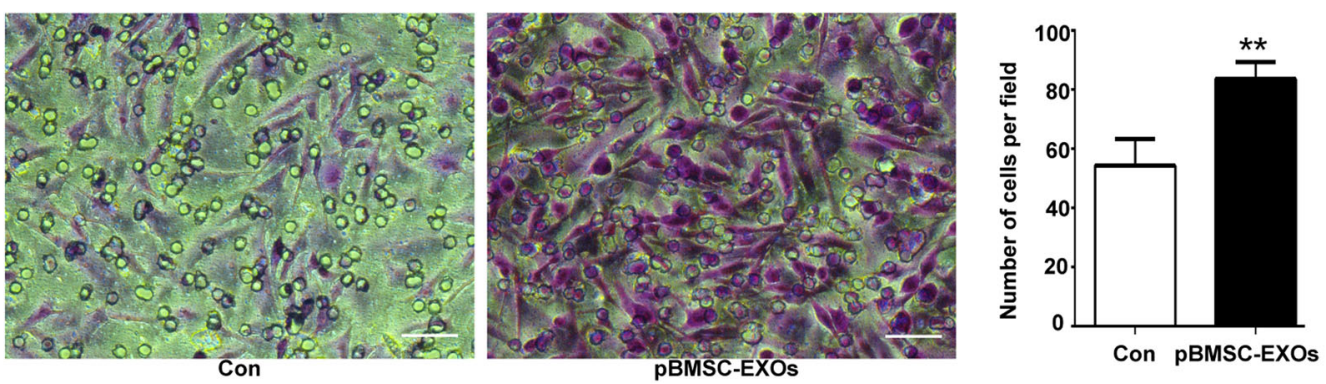

Fig. 4 Effects of the PBMSC-EXOs on the migration and proliferation of chondrocytes. A The proliferation of chondrocytes in coculture with pBMSCs and BMSCs. B Western blot analysis of the exosome-specific CD9, CD63, and TSG101 proteins. C Morphology of the exosomes under transmission electron microscopy. Scale bars, $100 \mathrm{~nm}$. D Wound-healing assays. E pBMSC-EXOs stimulated the migration of chondrocytes in the coculture system. Scale bars, $50 \mu \mathrm{m}$.

and the synthesis capacity of the chondrocytes adjacent to the injured site is limited ${ }^{34}$. Currently, BMSCs, adipose tissue-derived mesenchymal stem cells ${ }^{35}$, synovial mesenchymal stem cells ${ }^{36}$, and embryonic mesenchymal stem cells ${ }^{37}$ can be used as seed cells for tissue-engineered cartilage repair. Here, we demonstrated that pBMSCs 


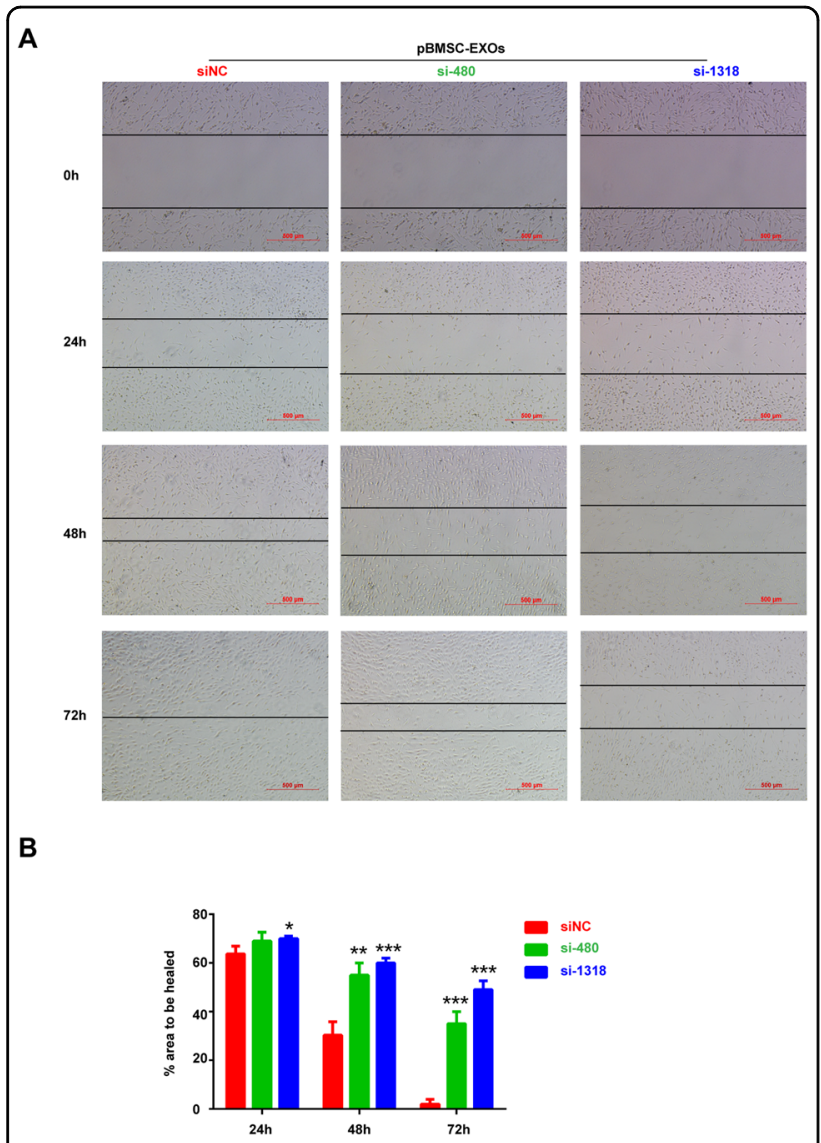

Fig. 5 pBMSC-EXOs inhibit the migration of chondrocytes upon BMP4 knockdown. A Light microscopy images of wound-healing area in cultures of chondrocytes with BMP4 knocked down. Scale bars, $500 \mu \mathrm{m}$. B Quantitative analysis of the migration rates at 24,48 , and $72 \mathrm{~h}$.

have a great ability to differentiate into chondrocytes. The differentiation of stem cells into chondrocytes is a complex process regulated by multiple genes. Sox9 is an important early transcription factor for chondrogenesis and chondrocyte differentiation, and it is a sensitive factor in articular cartilage tissue engineering, which plays an important role in chondrogenesis ${ }^{38,39}$. Collagen type II (COL2A1), as a downstream target protein Sox9, is an important component of the cartilage matrix and is thought to be the basis of structural strength against outside pressure ${ }^{40}$. Similarly, aggrecan (Acan) is one of the main components of the ECM of articular cartilage and is necessary to maintain the normal shape of cartilage tissue $^{41}$. In our study, we found that, compared with the levels in the BMSCs, the expression levels of COL2A1 and Acan were higher in the pBMSCs 14 days after chondrogenic differentiation, findings that confirm the predominance of the chondrogenic differentiation ability of pBMSCs.
In recent years, increasing evidence has suggested that stem cells and precursor cells may play important roles in promoting tissue regeneration by activating surrounding cells through paracrine mechanisms ${ }^{42,43}$. Most cells produce exosomes and play crucial roles in intercellular communication $^{44}$. Moreover, exosomes have been shown to have biological functions similar to that of the cells from which they were derived, and direct use of these nanoparticles has no obvious adverse effects, such as immune rejection or tumorigenicity ${ }^{45,46}$. In addition, exosomes can also promote the proliferation of chondrocytes in vitro $^{28}$. In this study, we used polydactyly tissue-derived BMSCs as "factories" to produce exosomes and found that pBMSC-EXOs enhanced the proliferation and migration of chondrocytes in vitro. However, pBMSC-EXOs did not affect the proliferation or migration of the FLSs from the OA patients, although FLSs are the main cells that mediate joint damage by secreting metalloproteinases. Previous studies have demonstrated that FLSs can induce osteoclastogenesis to promote bone absorption or release chemokines to recruit white blood cells to the joint, stimulating angiogenesis, which plays an important role in the initial phase of synovitis ${ }^{47}$. These results indicate that pBMSC-EXOs can promote the proliferation and migration of chondrocytes in vitro without causing adverse effects induced by the abnormal proliferation of synovial fibroblasts. In addition, we also confirmed that pBMSCs-EXOs led to a positive remission effect in the OA model, which was consistent with the previous protective effect shown with other types of stem cell-derived exosomes on OA models.

A variety of key signals, such as those from WNT, BMP, FGF, $\mathrm{HH}$, etc. were involved in the process of chondrocyte induction and differentiation ${ }^{48}$. For example, WNT5A and WNT11 play important regulatory roles in the chondrogenic differentiation of $\mathrm{MSCs}^{49}$. In addition, BMP2 or BMP4 can promote the differentiation of human embryonic stem cells into chondrocytes, leading to increased expression of chondrogenic genes Sox5, ACAN and COL2A $1^{50}$. Similarly, in this study, we found that BMP4 was highly expressed in the pBMSCs. Moreover, the pBMSCs transfected with BMP4 siRNAs were rarely able to differentiate into chondrocytes or promote the proliferation of chondrocytes in vitro. Therefore, BMP4 signaling plays an important role in the differentiation and proliferation of pBMSCs to chondrocytes in vitro.

In conclusion, pBMSC-EXOs promote not only the proliferation and migration of chondrocytes in vitro but also cartilage repair and delayed progression of $\mathrm{OA}$ in vivo. Therefore, $\mathrm{pBMSC}$-EXOs are expected not only to resolve the bottleneck of low chondrocyte regeneration and poor repair capacity but also to show great potential 
A

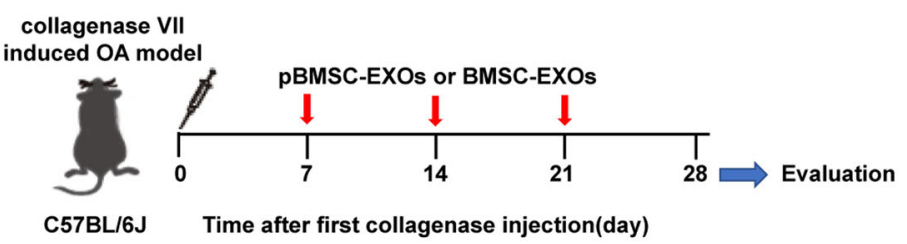

B
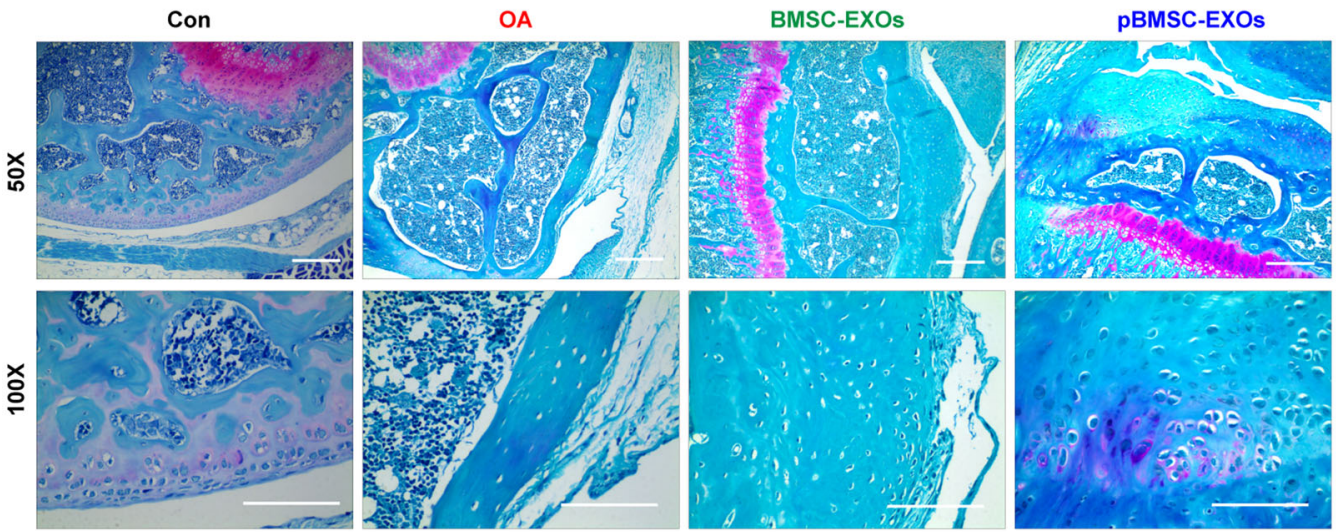

C

D

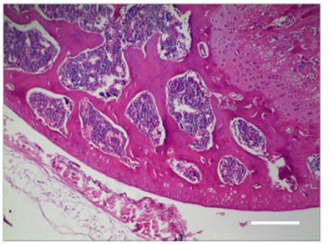

Con

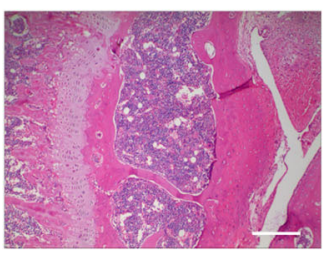

BMSC-EXOs

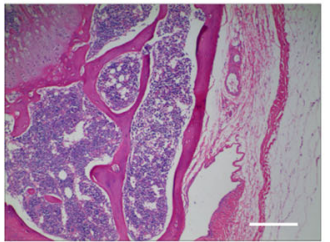

OA

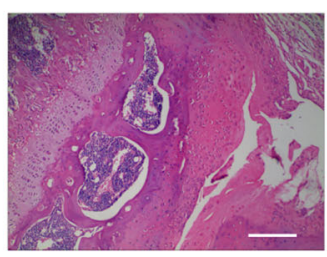

pBMSC-EXOs

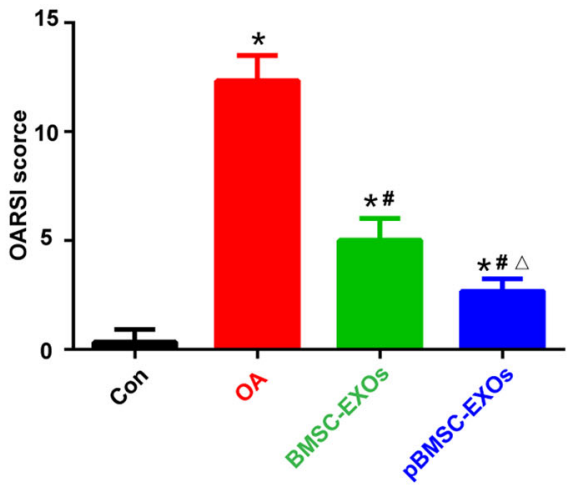

Fig. 6 Exosomes secreted by the pBMSCs for the treatment of osteoarthritis. A Flowchart of the in vivo experiment. B Safranin O/fast green staining. Scale bars, $100 \mu \mathrm{m}$. C H\&E staining. Scale bars, $100 \mu \mathrm{m}$. D OARSI scores of the OA, BMSC-EXO, and pBMSC-EXO groups were significantly higher than those of the control (Con) group, ${ }^{* *} P<0.01$. The scores of the BMSC-EXO and pBMSC-EXO groups were significantly lower than those of the OA group, ${ }^{\# \#} P<0.01$. Furthermore, there was a significant difference between the BMSC-EXO and pBMSC-EXO groups, $\triangle P<0.05$.

and good prospects for transformation and application in the treatment of injuries or defects of articular cartilage.

\section{Acknowledgements}

This work was supported by the project of the Jiangsu Provincial Medical Youth Talent (QNRC2016770), the Natural Science Foundation of the Jiangsu
Higher Education Institutions of China (19KJB320006), the Natural Science Foundation of Jiangsu Province (BK20160349 and BK20190173), the Science and Technology Project of Suzhou (SYS201761 and KJXW2019002), the National Natural Science Foundation of China (31500718, 82000157 and 81701596), and a Project Funded by the Priority Academic Program Development of Jiangsu Higher Education Institutions. 


\section{Author details}

${ }^{1}$ Institute of Pediatrics, Children's Hospital of Soochow University, Suzhou, Jiangsu, China. ${ }^{2}$ Department of Human Anatomy, Histology and Embryology, School of Biology and Basic Medical Sciences, Soochow University, Suzhou, Jiangsu, China. ${ }^{3}$ Jiangsu Institute of Clinical Immunology, The First Affiliated Hospital of Soochow University, Suzhou, Jiangsu, China. ${ }^{4}$ Institute of Blood and Marrow Transplantation, The First Affiliated Hospital of Soochow University, Suzhou, Jiangsu, China

\section{Conflict of interest}

The authors declare that they have no conflict of interest.

\section{Publisher's note}

Springer Nature remains neutral with regard to jurisdictional claims in published maps and institutional affiliations.

The online version of this article (https://doi.org/10.1038/s41420-020-00374-z) contains supplementary material, which is available to authorized users.

Received: 22 July 2020 Revised: 12 October 2020 Accepted: 9 November 2020

Published online: 10 December 2020

\section{References}

1. Loeser, R. F., Goldring, S. R., Scanzello, C. R. \& Goldring, M. B. Osteoarthritis: a disease of the joint as an organ. Arthritis Rheum. 64, 1697-1707 (2012).

2. Mathiessen, A. \& Conaghan, P. G. Synovitis in osteoarthritis: current understanding with therapeutic implications. Arthritis Res. Ther. 19, 18 (2017).

3. Roque, V., Agre, M., Barroso, J. \& Brito, I. Managing knee ostheoarthritis: efficacy of hyaluronic acid injections. Acta Reumatol. Port. 38, 154-161 (2013).

4. Liu-Bryan, R. \& Terkeltaub, R. Emerging regulators of the inflammatory process in osteoarthritis. Nat. Rev. Rheumatol. 11, 35-44 (2015).

5. van Meurs, J. B. \& Uitterlinden, A. G. Osteoarthritis year 2012 in review: genetics and genomics. Osteoarthr. Cartil. 20, 1470-1476 (2012).

6. Gao, B. et al. Melatonin rescued interleukin 1beta-impaired chondrogenesis of human mesenchymal stem cells. Stem Cell Res. Ther. 9, 162 (2018).

7. Wang, Y. et al. TNF-alpha-induced LRG1 promotes angiogenesis and mesenchymal stem cell migration in the subchondral bone during osteoarthritis. Cell Death Dis. 8, e2715 (2017).

8. Zhen, G. et al. Inhibition of TGF-beta signaling in mesenchymal stem cells of subchondral bone attenuates osteoarthritis. Nat. Med. 19, 704-712 (2013).

9. Bornes, T. D., Adesida, A. B. \& Jomha, N. M. Mesenchymal stem cells in the treatment of traumatic articular cartilage defects: a comprehensive review. Arthritis Res. Ther. 16, 432 (2014).

10. Frisbie, D. D., Kisiday, J. D., Kawcak, C. E., Werpy, N. M. \& Mcllwraith, C. W. Evaluation of adipose-derived stromal vascular fraction or bone marrowderived mesenchymal stem cells for treatment of osteoarthritis. J. Orthop. Res. 27, 1675-1680 (2009).

11. Zemljic, M., Pejkovic, B., Krajnc, I. \& Kocbek, L. Modern stem cell therapy: approach to disease. Wien. Klin. Wochenschr. 127 Suppl 5, S199-S203 (2015).

12. van Niel, G., D'Angelo, G. \& Raposo, G. Shedding light on the cell biology of extracellular vesicles. Nat. Rev. Mol. Cell Biol. 19, 213-228 (2018).

13. Colombo, M., Raposo, G. \& Thery, C. Biogenesis, secretion, and intercellular interactions of exosomes and other extracellular vesicles. Annu. Rev. Cell Dev. Biol. 30, 255-289 (2014)

14. Raposo, G.\& Stoorvogel, W. Extracellular vesicles: exosomes, microvesicles, and friends. J. Cell Biol. 200, 373-383 (2013).

15. Bobis-Wozowicz, S. et al. Human induced pluripotent stem cell-derived microvesicles transmit RNAs and proteins to recipient mature heart cells modulating cell fate and behavior. Stem Cells 33, 2748-2761 (2015).

16. Wang, Y. et al. Exosomes/microvesicles from induced pluripotent stem cells deliver cardioprotective miRNAs and prevent cardiomyocyte apoptosis in the ischemic myocardium. Int J. Cardiol. 192, 61-69 (2015).

17. Lee, $\mathrm{C}$. et al. Exosomes mediate the cytoprotective action of mesenchymal stromal cells on hypoxia-induced pulmonary hypertension. Circulation 126, 2601-2611 (2012).
18. Zhu, Y. G. et al. Human mesenchymal stem cell microvesicles for treatment of Escherichia coli endotoxin-induced acute lung injury in mice. Stem Cells $\mathbf{3 2}$ 116-125 (2014).

19. Farber, D. B. \& Katsman, D. Embryonic stem cell-derived microvesicles: could they be used for retinal regeneration? Adv. Exp. Med. Biol. 854, 563-569 (2016).

20. Xin, $\mathrm{H}$. et al. MiR-133b promotes neural plasticity and functional recovery after treatment of stroke with multipotent mesenchymal stromal cells in rats via transfer of exosome-enriched extracellular particles. Stem Cells 31, 2737-2746 (2013).

21. Gatti, S. et al. Microvesicles derived from human adult mesenchymal stem cells protect against ischaemia-reperfusion-induced acute and chronic kidney injury. Nephrol. Dial. Transplant. 26, 1474-1483 (2011).

22. Safdar, A. \& Tarnopolsky, M. A. Exosomes as mediators of the systemic adaptations to endurance exercise. Cold Spring Harb. Perspect. Med. 8, https:// doi.org/10.1101/cshperspect.a029827 (2018).

23. Sun, Q. et al. Amniotic fluid stem cells provide considerable advantages in epidermal regeneration: B7H4 creates a moderate inflammation microenvironment to promote wound repair. Sci. Rep. 5, 11560 (2015).

24. Comer, G. C., Potter, M. \& Ladd, A. L. Polydactyly of the hand. J. Am. Acad. Orthop. Surg. 26, 75-82 (2018).

25. Lin, Z., He, H., Wang, M. \& Liang, J. MicroRNA-130a controls bone marrow mesenchymal stem cell differentiation towards the osteoblastic and adipogenic fate. Cell Prolif. 52, e12688 (2019).

26. Thery, C., Amigorena, S., Raposo, G. \& Clayton, A. Isolation and characterization of exosomes from cell culture supernatants and biological fluids. Curr. Protoc. Cell Biol. Chapter 3, Unit 322 (2006).

27. Gurunathan, S., Kang, M. H., Jeyaraj, M., Qasim, M. \& Kim, J. H. Review of the isolation, characterization, biological function, and multifarious therapeutic approaches of exosomes. Cells 8, https://doi.org/10.3390/cells8040307 (2019).

28. Zhu, Y. et al. Comparison of exosomes secreted by induced pluripotent stem cell-derived mesenchymal stem cells and synovial membrane-derived mesenchymal stem cells for the treatment of osteoarthritis. Stem Cell Res. Ther. 8, 64 (2017).

29. min, Z. et al. Asymmetrical methyltransferase PRMT3 regulates human mesenchymal stem cell osteogenesis via miR-3648. Cell Death Dis. 10, 581 (2019).

30. Glasson, S. S., Chambers, M. G., Van Den Berg, W. B. \& Little, C. B. The OARSI histopathology initiative - recommendations for histological assessments of osteoarthritis in the mouse. Osteoarthr. Cartil. 18 Suppl 3, S17-S23 (2010).

31. Islam, M. N. et al. Mitochondrial transfer from bone-marrow-derived stromal cells to pulmonary alveoli protects against acute lung injury. Nat. Med. 18 759-765 (2012).

32. Ding, J., Wang, $X$. ., Chen, B., Zhang, J. \& Xu, J. Exosomes derived from human bone marrow mesenchymal stem cells stimulated by deferoxamine accelerate cutaneous wound healing by promoting angiogenesis. Biomed. Res. Int. 2019, 9742765 (2019).

33. Zhang, Q. et al. Comparison of therapeutic effects of different mesenchymal stem cells on rheumatoid arthritis in mice. PeerJ 7, e7023 (2019).

34. Krampera, M., Pizzolo, G., Aprili, G. \& Franchini, M. Mesenchymal stem cells for bone, cartilage, tendon and skeletal muscle repair. Bone 39, 678-683 (2006).

35. Zheng, D. et al. Controlled chondrogenesis from adipose-derived stem cells by recombinant transforming growth factor-beta3 fusion protein in peptide scaffolds. Acta Biomater. 11, 191-203 (2015).

36. Gale, A. L., Linardi, R. L., McClung, G., Mammone, R. M. \& Ortved, K. F. Comparison of the chondrogenic differentiation potential of equine synovial membrane-derived and bone marrow-derived mesenchymal stem cells. Front. Vet. Sci. 6, 178 (2019).

37. Wang, $Y$. et al. Exosomes from embryonic mesenchymal stem cells alleviate osteoarthritis through balancing synthesis and degradation of cartilage extracellular matrix. Stem Cell Res. Ther. 8, 189 (2017).

38. Zhang, H., Zhao, X., Zhang, Z., Chen, W. \& Zhang, X. An immunohistochemistry study of Sox9, Runx2, and Osterix expression in the mandibular cartilages of newborn mouse. Biomed. Res. Int. 2013, 265380 (2013).

39. Soltz, M. A., Basalo, I. M. \& Ateshian, G. A. Hydrostatic pressurization and depletion of trapped lubricant pool during creep contact of a rippled indenter against a biphasic articular cartilage layer. J. Biomech. Eng. 125, 585-593 (2003).

40. Zwickl, $\mathrm{H}$. et al. Correlation analysis of SOX9, -5 , and -6 as well as COL2A1 and aggrecan gene expression of collagen I implant-derived and osteoarthritic chondrocytes. Cartilage 7, 185-192 (2016).

41. Serrano, R. L., Chen, L. Y., Lotz, M. K., Liu-Bryan, R. \& Terkeltaub, R. Impaired proteasomal function in human osteoarthritic chondrocytes can contribute to 
decreased levels of SOX9 and aggrecan. Arthritis Rheumatol. 70, 1030-1041 (2018).

42. Kim, J. Y. et al. Human cord blood-derived endothelial progenitor cells and their conditioned media exhibit therapeutic equivalence for diabetic wound healing. Cell Transpl. 19, 1635-1644 (2010).

43. Zhang, M., Malik, A. B. \& Rehman, J. Endothelial progenitor cells and vascular repair. Curr. Opin. Hematol. 21, 224-228 (2014).

44. Thery, C., Ostrowski, M. \& Segura, E. Membrane vesicles as conveyors of immune responses. Nat. Rev. Immunol. 9, 581-593 (2009).

45. Burger, D. et al. Human endothelial colony-forming cells protect against acute kidney injury: role of exosomes. Am. J. Pathol. 185, 2309-2323 (2015).

46. Xin, H., Li, Y. \& Chopp, M. Exosomes/miRNAs as mediating cell-based therapy of stroke. Front. Cell. Neurosci. 8, 377 (2014).
47. Cai, S. et al. Similar transition processes in synovial fibroblasts from rheumatoid arthritis and osteoarthritis: a single-cell study. J. Immunol. Res. 2019, 4080735 (2019).

48. Fischer, J., Knoch, N., Sims, T., Rosshirt, N. \& Richter, W. Time-dependent contribution of BMP, FGF, IGF, and $\mathrm{HH}$ signaling to the proliferation of mesenchymal stroma cells during chondrogenesis. J. Cell Physiol. 233, 8962-8970 (2018)

49. Diederichs, S. et al. Regulation of WNT5A and WNT11 during MSC in vitro chondrogenesis: WNT inhibition lowers BMP and hedgehog activity, and reduces hypertrophy. Cell Mol. Life Sci. 76, 3875-3889 (2019).

50. Wang, T. et al. Enhanced chondrogenesis from human embryonic stem cells. Stem Cell Res. 39, 101497 (2019). 